\title{
The Fluctuating Two-Ray Fading Model for mmWave Communications
}

\author{
Juan M. Romero-Jerez*, F. Javier Lopez-Martinez ${ }^{+}$, José F. Paris ${ }^{+}$and Andrea Goldsmith ${ }^{\times}$ \\ *Dpto. Tecnología Electrónica, E.T.S.I. de Telecomunicación, \\ Universidad de Málaga, Campus de Excelencia Internacional Andalucia Tech, E-29071, Málaga (Spain) \\ ${ }^{+}$Dpto. Ingeniería de Comunicaciones, E.T.S.I. de Telecomunicación, \\ Universidad de Málaga, Campus de Excelencia Internacional Andalucia Tech, E-29071, Málaga (Spain) \\ ${ }^{\times}$Wireless Systems Lab, Dept. Electrical Engineering \\ Stanford University, Packard 371, 94305-9515 Stanford, CA. \\ romero@dte.uma.es, \{fjlopezm,paris\}@ic.uma.es, andrea@wsl.stanford.edu
}

\begin{abstract}
We introduce the Fluctuating Two-Ray (FTR) fading model, a new statistical channel model that consists of two fluctuating specular components with random phases plus a diffuse component. The FTR model arises as a natural generalization of the two-wave with diffuse power (TWDP) fading model proposed by Durgin, Rappaport and de Wolf; in this extended model, the two specular components exhibit a random amplitude fluctuation. Unlike in the TWDP model, we show that all the chief probability functions of the FTR fading model (PDF, CDF and MGF) can be expressed in closed-form. We also show that the FTR fading model provides a much better fit than the Rician fading model for recent small-scale fading measurements of the $28 \mathrm{GHz}$ outdoor millimeter-wave channels.
\end{abstract}

\section{INTRODUCTION}

There is much current interest in the use of millimeterwave (mmWave) bands as a means to overcome the wireless spectrum shortage caused by the exponential increase in aggregate traffic, particularly in the emerging standard of 5G [1]. As a result, research in mmWave radio communications has been boosted in the last years, particularly for its use in urban outdoor environments [2].

Understanding the behavior of the radio channel at mmWave bands is crucial for the development of transmission and reception strategies. As a result, research on channel modeling for mmWave wireless communications has been very intense in the recent years both in industry and academia [3-6]. Most of the stochastic channel models for mmWave communications use Rayleigh and Rician distributions to model the smallscale fading path amplitudes in NLOS and LOS scenarios, respectively. Very recently [7], the small-scale fading statistics obtained from a $28 \mathrm{GHz}$ outdoor measurement campaign showed that Rician fading was more suited than Rayleigh even in NLOS environments. However, a deeper look into the results of [7] indicates that conventional fading models in the literature fall short in accurately modeling the random fluctuations suffered by the received signal. In fact, the empirical CDFs [7] and PDFs [8] exhibit a bimodality that cannot be captured with existing generalized fading models $[9,10]$.
We here propose a new model to capture this behavior: the Fluctuating Two-Ray (FTR) fading model. This model is wellsuited to recreate the wide heterogeneity of random fluctuations that affect the mmWave radio signal when propagating in the presence of multiple scatterers. The FTR model is a natural generalization ${ }^{1}$ of the two-wave with diffuse power (TWDP) fading model proposed by Durgin, Rappaport and de Wolf [12]. It generalizes this model by allowing the constantamplitude specular waves associated to LOS propagation to randomly fluctuate. The inclusion of an additional source of randomness allows for a better characterization of the fluctuations suffered by the radio signal, compared to the TWDP model (which is a special case of the FTR model). Remarkably, this larger flexibility does not come at the price of an increased mathematical complexity, but instead facilitates a simpler statistical characterization than the TWDP model.

The benefits of using the FTR, which will be later exemplified, can be summarized as follows: (1) Despite being more general than the original TWDP model, its chief probability functions (CDF, PDF and MGF) are given in closed-form; (2) The FTR fading distribution is inherently bimodal, but also includes classical unimodal fading models like Rician or Rayleigh as special cases; and (3) The FTR fading distribution provides a much better fit than Rician fading to the $28 \mathrm{GHz}$ field measurements recently reported in [7].

The remainder of this paper is structured as follows: the physical justification of the FTR fading model is introduced in Section II. Then, in Section III, the statistical characterization of the FTR fading model is carried out. The empirical validation of our model is presented in Section IV by fitting the FTR fading model to small-scale fading field measurements in the mmWave band. Finally, the main conclusions are drawn in Section V.

\footnotetext{
${ }^{1} \mathrm{We}$ also note that the FTR model here proposed differs from the Generalized Two-Ray (GTR) model proposed in [11]. Unlike the TWDP model, the GTR model allows the phase distributions of the specular waves to be other than uniform, but the amplitudes of the specular components is still kept constant.
} 


\section{Preliminaries AND Channel Model}

The rapid variations of mutipath components in wireless channels leads to dramatic fluctuations in the received signal strength, which is usually referred to as multipath (or smallscale) fading. The small-scale fluctuations of the wireless channel are typically modeled by a set of $N$ dominant waves, referred to as specular components, to which other diffusely propagating waves are added [12]. Thus, the complex baseband voltage of a wireless channel experiencing multipath fading can be expressed as

$$
V_{r}=\sum_{n=1}^{N} V_{n} \exp \left(j \phi_{n}\right)+X+j Y,
$$

where $V_{n} \exp \left(j \phi_{n}\right)$ represents the $n$-th specular component, which is assumed to have a constant amplitude $V_{n}$ and a uniformly distributed random phase $\phi_{n}$, such that $\phi_{n} \sim \mathcal{U}[0,2 \pi)$. Since the distance traversed by the propagating waves are typically orders of magnitude greater than their wavelengths, the random phase variables can be considered statistically independent. On the other hand, $X+j Y$ is a complex Gaussian random variable, such that $X, Y \sim \mathcal{N}\left(0, \sigma^{2}\right)$, representing the diffuse received signal component due to the combined reception of numerous weak, independently-phased scattered waves for which the central limit theorem can be applied.

The general model presented in (1) includes very important statistical wireless channel models as particular cases. Thus, when $N=0$, i.e., no specular component is present, the Rayleigh fading model is obtained, while for $N=1$, a single dominant specular component, we have the Rician fading model. The case when there are two dominant specular components, $N=2$, is usually referred to as the Two Wave with Diffuse Power (TWDP) fading model or, alternatively, the Generalized Two-Ray with Uniformly distributed phases model (GTR-U) [11]. This model has received increased attention in the last few years, as it contains the aforementioned classical models as particular cases and it accurately fits field measurements in a variety of propagation scenarios [12]. Unfortunately, the statistical analysis of the TWDP fading model is much more complicated than in the case of the Rayleigh or Rician fading models, as there is not known expressions for either the PDF and the CDF of the received signal envelope.

Prior works have considered variations in the amplitude of the dominant specular components, often associated with the LOS propagation, under different scenarios, and this model has also been validated with field measurements. Examples include the Rician shadowed fading model [13] as a generalization of Rician fading model, and the $\kappa-\mu$ shadowed fading model introduced in [10] as a generalization of Yacoub's $\kappa-\mu$ fading model. However, while the word "shadowing" was used when the models $[10,13]$ were introduced, these models should not necessarily be linked to the large-scale fading phenomena called shadowing, caused by a complete or partial blockage by obstacles many times larger than the signal wavelength. Rather, the models in $[10,13]$ considered shadowing to be any amplitude fluctuation in the specular waves (e.g. due to shadowing by objects or human bodies, propagation condition variations or fast moving scatterers) that takes place over the time period of interest. Therefore, considering the amplitudes of the specular components to be modulated by a Nakagami$m$ random variable with squared unit mean as in $[10,13]$, we can write:

$$
V_{r}=\sum_{n=1}^{N} \sqrt{\zeta} V_{n} \exp \left(j \phi_{n}\right)+X+j Y,
$$

where $\zeta$ is a unit-mean Gamma distributed random variable with PDF

$$
f_{\zeta}(u)=\frac{m^{m} u^{m-1}}{\Gamma(m)} e^{-m u} .
$$

The wireless channel model given in (2)-(3) for the particular case when $N=1$ corresponds to the well-known Rician shadowed fading model [13]. In the rest of this paper, we will consider the case when $N=2$ and will derive a statistical description of the resulting channel model. This model will be subsequently denoted as the Fluctuating Two-Ray (FTR) model, in order to indicate the presence of two specular components with random phase for which their amplitude exhibits a random fluctuation.

\section{STATISTICAL CHARACTERIZATION OF THE FTR FADING MODEL}

Let us consider that the complex baseband received signal can be written as

$$
V_{r}=\sqrt{\zeta} V_{1} \exp \left(j \phi_{1}\right)+\sqrt{\zeta} V_{2} \exp \left(j \phi_{2}\right)+X+j Y .
$$

This model is conveniently expressed in terms of the parameters $K$ and $\Delta$, defined as

$$
\begin{aligned}
& K=\frac{V_{1}^{2}+V_{2}^{2}}{2 \sigma^{2}}, \\
& \Delta=\frac{2 V_{1} V_{2}}{V_{1}^{2}+V_{2}^{2}} .
\end{aligned}
$$

The $K$ parameter represents the ratio of the average power of the dominant components to the power of the remaining diffuse multipath, just like the Rician $K$ parameter. On the other hand, $\Delta$ is a parameter ranging from 0 to 1 expressing the similarity of the received powers from the specular components: when the magnitudes of the two specular components are equal, $\Delta=1$, while in the absence of a second component ( $V_{1}=0$ or $\left.V_{2}=0\right), \Delta=0$. Note that $\Delta=0$ yields the Rician fading model.

We will first characterize the distribution of the received power envelope associated with the FTR fading model, or equivalently, the distribution of the received signal-to-noise ratio (SNR). After passing through the multipath fading channel, the signal will be affected by additive white Gaussian noise (AWGN) with one-sided power spectral density $N_{0}$. The statistical characterization of the instantaneous SNR, here denoted as $\gamma$, is crucial for the analysis and design of wireless communications systems, as many performance metrics in wireless communications are a function of the SNR. 
The received average SNR $\bar{\gamma}$ after transmitting a symbol with energy density $E_{s}$ undergoing a multipath fading channel as described in (4) will be

$$
\begin{aligned}
\bar{\gamma}= & \left(E_{b} / N_{0}\right) \mathbb{E}\left\{\left|V_{r}\right|^{2}\right\}=\left(E_{b} / N_{0}\right)\left(V_{1}^{2}+V_{2}^{2}+2 \sigma^{2}\right) \\
= & \left(E_{b} / N_{0}\right) 2 \sigma^{2}(1+K),
\end{aligned}
$$

where $\mathbb{E}\{\cdot\}$ denotes the expectation operator.

With all the above definitions, the main probability functions in performance analysis under the FTR fading model can now be computed.

\section{A. $M G F$}

In the following lemma we show that, for the FTR fading model, it is possible to obtain the MGF of $\gamma$ in closed-form.

Lemma 1: Let us consider the FTR fading model as described in (4)-(7). Then, the MGF of the received SNR $\gamma$ will be given by

$$
\begin{aligned}
M_{\gamma}(s)= & \frac{m^{m}(1+K)(1+K-\bar{\gamma} s)^{m-1}}{(\sqrt{\mathcal{R}(m, k, \Delta ; s)})^{m}} \\
& \times P_{m-1}\left(\frac{m(1+K)-(m+K) \bar{\gamma} s}{\sqrt{\mathcal{R}(m, k, \Delta ; s)}}\right),
\end{aligned}
$$

where $\mathcal{R}(m, k, \Delta ; s)$ is a polynomial in $s$ defined as

$$
\begin{aligned}
& R(m, k, \Delta ; s)=\left[(m+K)^{2}-\Delta^{2} K^{2}\right] \bar{\gamma}^{2} s^{2} \\
& -2 m(1+K)(m+K) \bar{\gamma} s+m^{2}(1+K)^{2},
\end{aligned}
$$

and $P_{\mu}(\cdot)$ is the Legendre function of the first kind of degree $\mu$, which can be calculated as

$$
P_{\mu}(z)={ }_{2} F_{1}\left(-\mu, \mu+1 ; 1 ; \frac{1-z}{2}\right),
$$

given that $|1-z|<2$, where ${ }_{2} F_{1}(\cdot)$ is the Gauss hypergeometric function [14, p. 556 (15.1.1)].

Proof: Let us consider the fading channel model given in (4) conditioned to a particular realization, $\zeta=u$, of the random variable modeling the fluctuation of the specular components. In this case, we can write

$$
\left.V_{r}\right|_{\zeta=u}=\sqrt{u} V_{1} \exp \left(j \phi_{1}\right)+\sqrt{u} V_{2} \exp \left(j \phi_{2}\right)+X+j Y
$$

This corresponds to the classical TWDP fading model where the amplitudes of the specular components are given by $\sqrt{u} V_{1}$ and $\sqrt{u} V_{2}$, for which the following parameters can be defined:

$$
\begin{aligned}
& K_{u}=\frac{u V_{1}^{2}+u V_{2}^{2}}{2 \sigma^{2}}=u \frac{V_{1}^{2}+V_{2}^{2}}{2 \sigma^{2}}, \\
& \Delta_{u}=\frac{2 \sqrt{u} V_{1} \sqrt{u} V_{2}}{u V_{1}^{2}+u V_{2}^{2}}=\frac{2 V_{1} V_{2}}{V_{1}^{2}+V_{2}^{2}} .
\end{aligned}
$$

It is clear that these parameters are related to those defined in (5) and (6) for the FTR fading model by

$$
\begin{gathered}
K_{u}=u K, \\
\Delta_{u}=\Delta .
\end{gathered}
$$

The conditional average SNR for the fading model described in (11) will be

$$
\begin{aligned}
\bar{\gamma}_{u} & =\left(E_{b} / N_{0}\right)\left(u V_{1}^{2}+u V_{2}^{2}+2 \sigma^{2}\right) \\
& =\left(E_{b} / N_{0}\right) 2 \sigma^{2}\left(1+K_{u}\right) .
\end{aligned}
$$

The MGF of the TWDP fading model was shown in [11] to be given in closed-form as

$$
\begin{aligned}
M_{\gamma_{u}}(s)= & \frac{1+K_{u}}{1+K_{u}-\bar{\gamma}_{u} s} \exp \left(\frac{K_{u} \bar{\gamma}_{u} s}{1+K_{u}-\bar{\gamma}_{u} s}\right) \\
& \times I_{0}\left(\frac{\Delta_{u} K_{u} \bar{\gamma}_{u} s}{1+K_{u}-\bar{\gamma}_{u} s}\right),
\end{aligned}
$$

where $I_{0}(\cdot)$ is the zero-order modified Bessel function of the first kind. This MGF can be written in terms of the $K$ and $\Delta$ parameters defined for the FTR model. Note that from (7) and (16) we can write, respectively,

$$
\begin{aligned}
\frac{(1+K)}{\bar{\gamma}} & =\frac{1}{\left(E_{b} / N_{0}\right) 2 \sigma^{2}}, \\
\frac{\left(1+K_{u}\right)}{\bar{\gamma}_{u}} & =\frac{1}{\left(E_{b} / N_{0}\right) 2 \sigma^{2}},
\end{aligned}
$$

and equating (18) and (19) it is clear that

$$
\frac{\left(1+K_{u}\right)}{\bar{\gamma}_{u}}=\frac{(1+K)}{\bar{\gamma}} .
$$

Now, taking into account (14), (15) and (20), we have

$$
\begin{gathered}
M_{\gamma_{u}}(s)=\frac{\frac{1+K_{u}}{\bar{\gamma}_{u}}}{\frac{1+K_{u}}{\bar{\gamma}_{u}}-s} \exp \left(\frac{K_{u} s}{\frac{1+K_{u}}{\bar{\gamma}_{u}}-s}\right) \\
\times I_{0}\left(\Delta_{u} \frac{K_{u} s}{\frac{1+K_{u}}{\bar{\gamma}_{u}}-s}\right) \\
=\frac{\frac{1+K}{\bar{\gamma}}}{\frac{1+K}{\bar{\gamma}}-s} \exp \left(\frac{u K s}{\frac{1+K}{\bar{\gamma}}-s}\right) I_{0}\left(\Delta \frac{u K s}{\frac{1+K}{\bar{\gamma}}-s}\right),
\end{gathered}
$$

and therefore the conditional MGF can be written as

$$
M_{\gamma_{u}}(s)=\mathcal{B}(s) e^{u \mathcal{A}(s)} I_{0}(u \Delta \mathcal{A}(s)),
$$

where we have defined

$$
\mathcal{A}(s)=\frac{K \bar{\gamma} s}{1+K-\bar{\gamma} s}, \quad \mathcal{B}(s)=\frac{1+K}{1+K-\bar{\gamma} s} .
$$

The MGF of the SNR of the FTR model can be obtained by averaging (22) over all possible realizations $u$ of the random variable $\zeta$, i.e.,

$$
\begin{aligned}
& M_{\gamma}(s)=\int_{0}^{\infty} M_{\gamma_{u}}(s) f_{\zeta}(u) d u \\
& =B(s) \frac{m^{m}}{\Gamma(m)} \int_{0}^{\infty} u^{m-1} e^{-u(m-A(s))} I_{0}(u \Delta A(s)) d u .
\end{aligned}
$$

The integral in (24) can be solved in closed-form, as from [15, p. 196 (8)] we have

$$
\int_{0}^{\infty} t^{\mu} e^{-\beta t} I_{0}(\alpha t) d t=\Gamma(\mu+1) \theta^{-\mu-1} P_{\mu}(\beta / \theta),
$$




$$
\begin{aligned}
& f_{\gamma}(x)= \frac{1}{2^{m-1}} \frac{1+K}{\bar{\gamma}}\left(\frac{m}{\sqrt{(m+K)^{2}-K^{2} \Delta^{2}}}\right)^{m} \sum_{q=0}^{\lfloor(m-1) / 2\rfloor}(-1)^{q} C_{q}^{m-1}\left(\frac{m+K}{\sqrt{(m+K)^{2}-K^{2} \Delta^{2}}}\right) \\
& \times \Phi_{2}^{(4)}\left(1+2 q-m, m-q-\frac{1}{2}, m-q-\frac{1}{2}, 1-m ; 1 ;\right.\left.\quad-\frac{m(1+K)}{(m+K) \bar{\gamma}} x,-\frac{m(1+K)}{(m+K(1+\Delta)) \bar{\gamma}} x,-\frac{m(1+K)}{(m+K(1-\Delta)) \bar{\gamma}} x,-\frac{1+K}{\bar{\gamma}} x\right) . \\
& F_{\gamma}(x)=\frac{1}{2^{m-1}} \frac{1+K}{\bar{\gamma}}\left(\frac{m}{\sqrt{(m+K)^{2}-K^{2} \Delta^{2}}}\right)^{m} \sum_{q=0}^{m(m-1) / 2\rfloor}(-1)^{q} C_{q}^{m-1}\left(\frac{m+K}{\sqrt{(m+K)^{2}-K^{2} \Delta^{2}}}\right)^{m-1-2 q} \\
& \times x \Phi_{2}^{(4)}\left(1+2 q-m, m-q-\frac{1}{2}, m-q-\frac{1}{2}, 1-m ; 2 ; \quad \frac{m(1+K)}{(m+K)} x,-\frac{m(1+K)}{(m+K(1-\Delta)) \bar{\gamma}} x,-\frac{1+K}{\bar{\gamma}} x\right) .
\end{aligned}
$$

where $\theta=\sqrt{\beta^{2}-\alpha^{2}}$. Using (25) to solve (24), after some algebraic manipulations, (8) is obtained.

The FTR fading model introduced here is well-suited to recreate the propagation conditions in a wide variety of wireless scenarios, ranging from very favorable ones to worse-than Rayleigh fading. It also includes many important well-known statistical fading models as particular cases, i.e., TWDP, Rician shadowed, Rician, Rayleigh. one-sided Gaussian, Nakagami$m$ and Nakagami- $q$ (Hoyt). With the MGF in closed-form, we now show that the PDF and CDF of the FTR fading distribution can also be obtained in closed-form, provided that the parameter $m$ is restricted to take positive integer values (i.e., $m \in \mathbb{Z}^{+}$).

\section{B. $P D F$ and $C D F$}

When the parameter $m$ takes integer values, the MGF of the SNR in the FTR fading model can be calculated as a finite sum of elementary terms. This is based on the fact that, for $m$ an integer, the Legendre function in the MGF given in (8) has an integer degree, thus becoming a Legendre polynomial. A Legendre polynomial of degree $n$ can be written as $[14, \mathrm{p}$. 775 (22.3.8)]

$$
P_{n}(z)=\frac{1}{2^{n}} \sum_{q=0}^{\lfloor n / 2\rfloor}(-1)^{q} C_{q}^{n} z^{n-2 q},
$$

where $\lfloor\cdot\rfloor$ is the floor function and $C_{q}^{n}$ is a coefficient given by

$$
C_{q}^{n}=\left(\begin{array}{c}
n \\
q
\end{array}\right)\left(\begin{array}{c}
2 n-2 q \\
n
\end{array}\right)=\frac{(2 n-2 q) !}{q !(n-q) !(n-2 q) !} .
$$

Lemma 2: When $m \in \mathbb{Z}^{+}$, the PDF and CDF of the SNR $\gamma$ in a FTR fading channel can be expressed in terms of the confluent hypergeometric function $\Phi_{2}(\cdot)$ defined in [16, p. 34, (8)], as given, respectively, in (28) and (29).

Proof: We note that the polynomial $\mathcal{R}(m, k, \Delta ; s)$ defined in (9) can be factorized as

$$
\begin{gathered}
\mathcal{R}(m, K, \Delta ; s)=[m(1+K)-(m+K(1+\Delta)) \bar{\gamma} s] \\
\times[m(1+K)-(m+K(1-\Delta)) \bar{\gamma} s] .
\end{gathered}
$$

For the sake of compactness, let us define the following parameters;

$$
\begin{aligned}
& a_{1}=\frac{m(1+K)}{(m+K) \bar{\gamma}}, \quad a_{2}=\frac{m(1+K)}{(m+K(1+\Delta)) \bar{\gamma}}, \\
& a_{3}=\frac{m(1+K)}{(m+K(1-\Delta)) \bar{\gamma}}, \quad a_{4}=\frac{1+K}{\bar{\gamma}} .
\end{aligned}
$$

From (8), using (26) and (30), the MGF of $\gamma$ can be rewritten as

$$
\begin{aligned}
& M_{\gamma}(s)=\frac{-\left(a_{2} a_{3}\right)^{\frac{m}{2}}}{\left(2 a_{4}\right)^{m-1}} \sum_{q=0}^{\lfloor(m-1) / 2\rfloor}(-1)^{q} \\
& \times C_{q}^{m-1}\left(\frac{\left(a_{2} a_{3}\right)^{\frac{1}{2}}}{a_{1}}\right)^{m-1-2 q} \frac{1}{s}\left(1-\frac{a_{1}}{s}\right)^{m-1-2 q} \\
& \times\left(1-\frac{a_{2}}{s}\right)^{\frac{1}{2}+q-m}\left(1-\frac{a_{3}}{s}\right)^{\frac{1}{2}+q-m}\left(1-\frac{a_{4}}{s}\right)^{m-1} .
\end{aligned}
$$

Taking into account that the PDF is related to the MGF by the inverse Laplace transform, i.e., $f_{\gamma}(x)=\mathcal{L}^{-1}\left[M_{\gamma}(-s) ; x\right]$, (28) follows from (32) and the Laplace transform pair given in [17, eq. (4.24.3)]. On the other hand, (29) is obtained analogously by considering that $F_{\gamma}(x)=\mathcal{L}^{-1}\left[M_{\gamma}(-s) / s ; x\right]$.

We now provide some remarks on these derivations. First, despite requiring the evaluation of a confluent hypergeometric function, the PDF and CDF of the FTR fading model can be expressed in terms of a well-known function in communication 


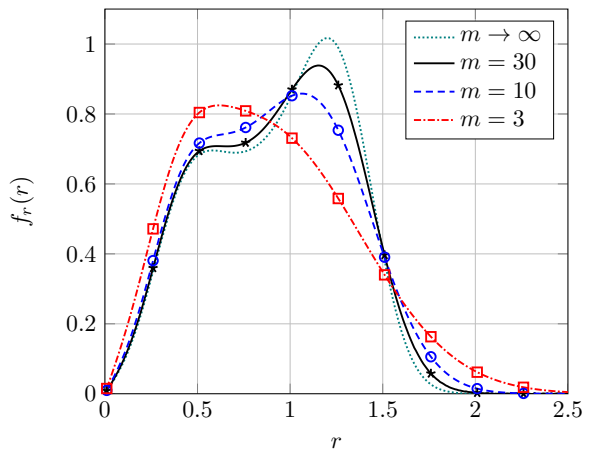

(a) Fixed $K=15, \Delta=0.9$

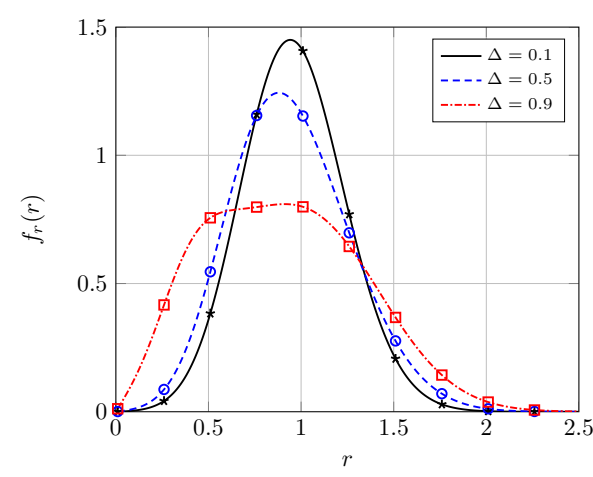

(b) Fixed $m=5, K=15$

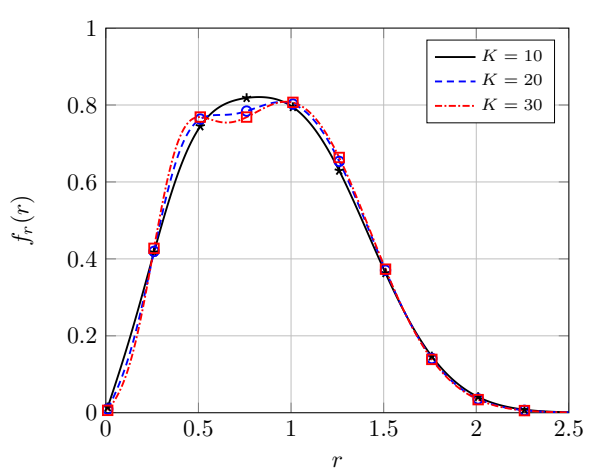

(c) Fixed $m=5, \Delta=0.9$

Fig. 1: Envelope PDF of the FTR fading model for different sets of parameter values and $\Omega=1$.

theory. In fact, the $\Phi_{2}$ function also makes an appearance in the CDF of simpler fading models such as Rician shadowed and $\kappa-\mu$ shadowed $[10,17]$. Note also that this function can be efficiently evaluated using an inverse Laplace transform as described in [18, Appendix 9B].

Secondly, the PDF and CDF of the received signal envelope $r$ can be easily derived from (28) and (29) by a simple change of variables. Specifically, $f_{r}(r)=2 r f_{\gamma}\left(r^{2}\right)$ and $F_{r}(r)=$ $F_{\gamma}\left(r^{2}\right)$, and replacing $\bar{\gamma}$ by $\Omega$, where $\Omega=E\left\{r^{2}\right\}$.

Finally, the FTR fading model exhibits a bimodal behavior for some choices of the parameters $K, \Delta$ and $m$. Similarly to the TWDP fading model, the bimodality is dominated by the parameters $K$ and $\Delta$. Specifically, for $\Delta$ approximately 1 and large values of $K$, the bimodality is more pronounced; this corresponds to the worse-than Rayleigh fading case. The additional parameter $m$ smooths the bimodality ${ }^{2}$ as $m$ decreases; conversely, as $m \rightarrow \infty$, the FTR fading model reduces to the TWDP fading model. The effect of the FTR model parameters on the bimodality of the distribution is exemplified in Fig. 1.

\section{EMPIRICAL VALIDATION}

In the previous sections, we have introduced the FTR fading model and derived its more relevant statistics. We will now show its suitability for modeling small-scale fading in mmWave wireless links based on empirical data matching. This close matching results from the fact that the FTR fading model allows for incorporating the effect of having a reduced number of scatterers by means of its two specular waves, while the incorporation of the amplitude fluctuation on such components gives an additional degree of freedom to the model. We use the empirical results presented in [7] to validate the FTR fading model in the context of small-scale fading modeling of mmWave outdoor communications in the $28 \mathrm{GHz}$ band. Details on the specific measurement configuration can be found in [7].

A modified version of the Kolmogorov-Smirnov (KS) statistic has been used to define the error factor $\epsilon$ used to quantify

\footnotetext{
${ }^{2}$ The bimodality of the distribution is clearly identified by the appearance of two maxima on its PDF; this would be translated into several transitions from concavity to convexity (i.e., inflection points) on the CDF.
}

the goodness of fit between the empirical and theoretical CDFs, denoted by $\hat{F}_{r}(\cdot)$ and $F_{r}(\cdot)$ respectively, i.e,

$$
\epsilon \triangleq \max _{x}\left|\log _{10} \hat{F}_{r}(x)-\log _{10} F_{r}(x)\right| .
$$

Note that the CDF is used in log-scale in order to outweigh the fit in those amplitude values closer to zero, where the fading is more severe [19].

In Figs. 2 and 3 we compare, respectively, the set of measurements corresponding to the LOS and NLOS crosspolarized scenarios described in [7, Fig. 6]. For this set of measurements, the empirical CDFs lie within the theoretical CDFs corresponding to a Rician distribution with values of $K$ ranging from 2 to 7 (i.e. 3 to $8 \mathrm{~dB}$ ). According to the $\mathrm{KS}$ statistic, the values of $K$ that provide the best fit to the Rician distribution are $K_{\mathrm{LOS}}^{\mathrm{Rice}}=4.04$ and $K_{\mathrm{NLOS}}^{\text {Rice }}=4.78$ respectively. Such values of $K$ yield an error factor value of $\epsilon_{\mathrm{LOS}}^{\text {Rice }}=0.3302$ and $\epsilon_{\mathrm{NLOS}}^{\text {Rice }}=0.3571$. Now, using the proposed FTR fading model, we obtain the following set of parameters for the LOS and NLOS cases: FTR $_{\mathrm{LOS}}=(K=80, \Delta=0.5873, m=2)$ and $\mathrm{FTR}_{\mathrm{NLOS}}=(K=32.7, \Delta=0.8331, m=10)$. Note that the parameter $m$ plays a key role in the goodness of fit, as it enables the CDF to modify its concavity and convexity in order to better adjust the empirical data. For these parameters, the error factor value obtained by the FTR fit are $\epsilon_{\mathrm{LOS}}^{\mathrm{FTR}}=0.2246$ and $\epsilon_{\mathrm{NLOS}}^{\mathrm{FTR}}=0.2681$. Thus, a remarkable improvement is attained when using the FTR fading model with respect to the Rician model.

\section{Conclusions}

A new model for small-scale fading, the FTR fading model, was introduced. Based on a physical justification that characterizes the random fluctuations on the dominant specular components of the received signal, the FTR fading model is shown to be a promising candidate for modeling small-scale fading in the context of mmWave communications because it offers a good balance between versatility, flexibility, goodness of fit and physical underpinnings. The fit to empirical measurements in the $28 \mathrm{GHz}$ band shows great improvements over the Rician fading model. 


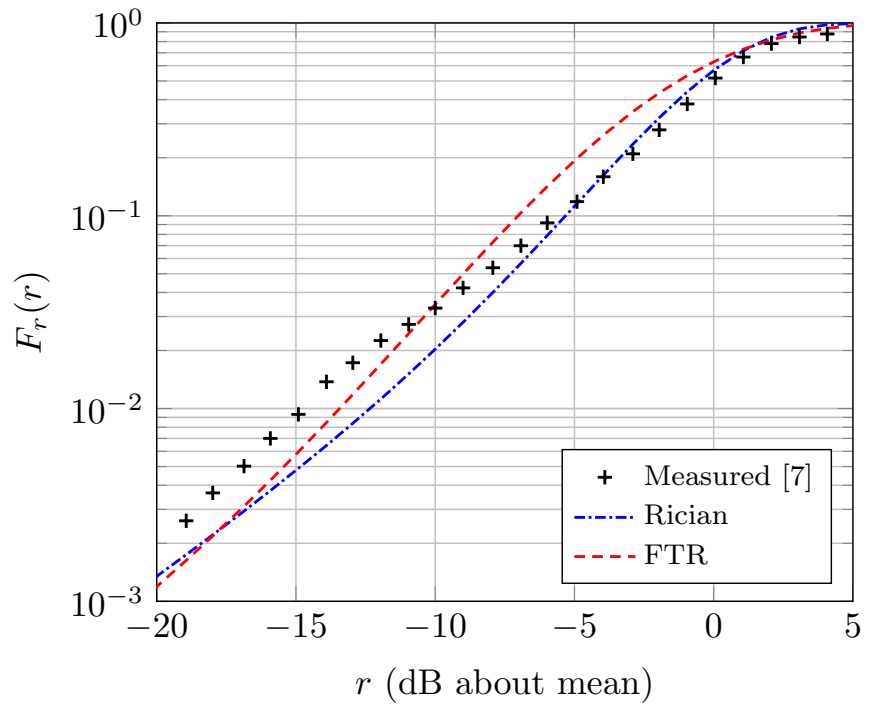

Fig. 2: Empirical vs theoretical CDFs of the received signal amplitude for LOS scenario. Parameter values are $K_{\text {Rice }}=4.04$ and $K_{\mathrm{FTR}}=80, \Delta=0.5873, m=2$. Measured data obtained from [7, Fig. 6, LOS].

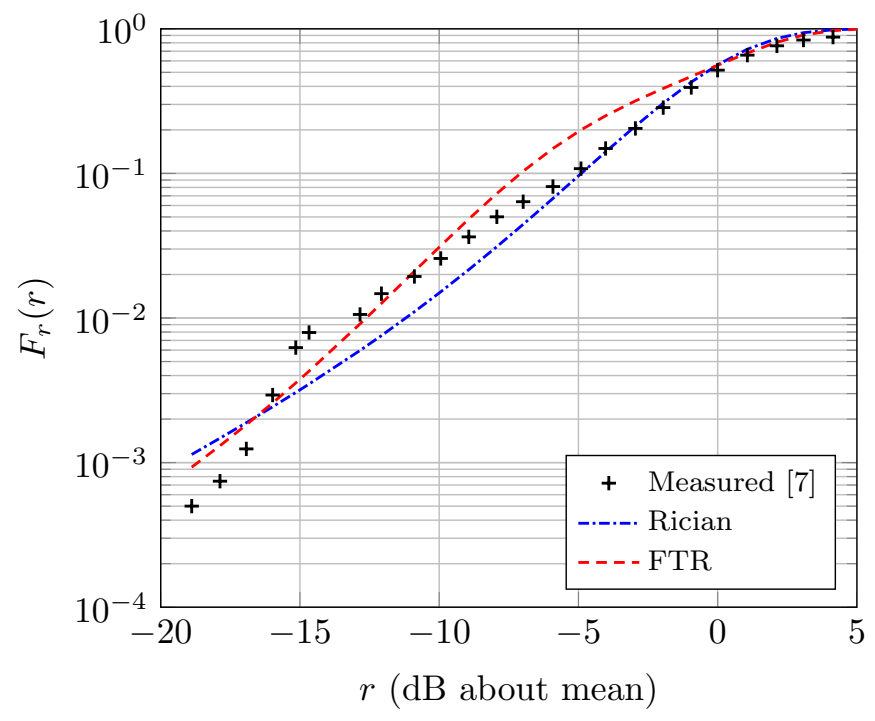

Fig. 3: Empirical vs theoretical CDFs of the received signal amplitude for NLOS scenario Parameter values are $K_{\text {Rice }}=4.78$ and $K_{\mathrm{FTR}}=32.7, \Delta=0.8331, m=10$. Measured data obtained from [7, Fig. 6, NLOS].

\section{ACKNOWLEDGMENT}

This work has been funded by Universidad de Málaga (Plan Propio), the Consejería de Economía, Innovación, Ciencia y Empleo of the Junta de Andalucía, the Spanish Government and the European FEDER projects P2011-TIC-7109, P2011-TIC-8238, TEC2014-57901-R, TEC2013-42711-R and TEC2013-44442-P.

\section{REFERENCES}

[1] F. Boccardi, R. W. Heath, A. Lozano, T. L. Marzetta, and P. Popovski, "Five disruptive technology directions for 5G," IEEE Communications Magazine, vol. 52, no. 2, pp. 74-80, February 2014.

[2] T. S. Rappaport, S. Sun, R. Mayzus, H. Zhao, Y. Azar, K. Wang, G. N. Wong, J. K. Schulz, M. Samimi, and F. Gutierrez, "Millimeter Wave Mobile Communications for 5G Cellular: It Will Work!" IEEE Access, vol. 1, pp. 335-349, 2013.

[3] M. R. Akdeniz, Y. Liu, M. K. Samimi, S. Sun, S. Rangan, T. S. Rappaport, and E. Erkip, "Millimeter Wave Channel Modeling and Cellular Capacity Evaluation," IEEE Journal on Selected Areas in Communications, vol. 32, no. 6, pp. 1164-1179, June 2014.

[4] J. Kyröläinen, P. Kyösti, J. Meinilä, V. Nurmela, L. Raschkowski, A. Roivainen, and J. Ylitalo, "Channel modelling for the fifth generation mobile communications," in Proc. 8th European Conference on Antennas and Propagation, EuCAP 2014.

[5] T. S. Rappaport, G. R. MacCartney, M. K. Samimi, and S. Sun, "Wideband Millimeter-Wave Propagation Measurements and Channel Models for Future Wireless Communication System Design," IEEE Trans. Comm., vol. 63, no. 9, pp. 3029-3056, Sept 2015.

[6] S. Hur, S. Baek, B. Kim, Y. Chang, A. F. Molisch, T. S. Rappaport, K. Haneda, and J. Park, "Proposal on Millimeter-Wave Channel Modeling for 5G Cellular System," IEEE Journal of Selected Topics in Signal Processing, vol. 10, no. 3, pp. 454-469, April 2016.

[7] M. K. Samimi and T. S. Rappaport, " $28 \mathrm{GHz}$ Millimeter-Wave Ultrawideband Small-Scale Fading Models in Wireless Channels," arXiv preprint arXiv:1511.06938, 2015.

[8] T. Mavridis, L. Petrillo, J. Sarrazin, A. Benlarbi-Delaï, and P. D. Doncker, "Near-Body Shadowing Analysis at $60 \mathrm{GHz}$," IEEE Transactions on Antennas and Propagation, vol. 63, no. 10, pp. 4505-4511, Oct 2015.

[9] M. Yacoub, "The $\kappa-\mu$ distribution and the eta- $\mu$ distribution," IEEE Antennas and Propagation Magazine, vol. 49, no. 1, pp. 68-81, Feb 2007.

[10] J. F. Paris, "Statistical Characterization of $\kappa-\mu$ Shadowed Fading," IEEE Trans. Veh. Technol., vol. 63, no. 2, pp. 518-526, Feb 2014.

[11] M. Rao, F. Lopez-Martinez, M.-S. Alouini, and A. Goldsmith, "MGF Approach to the Analysis of Generalized Two-Ray Fading Models," IEEE Trans. Wireless Commun., vol. 14, no. 5, pp. 2548-2561, May 2015.

[12] G. D. Durgin, T. S. Rappaport, and D. A. de Wolf, "New analytical models and probability density functions for fading in wireless communications," IEEE Trans. Comm., vol. 50, no. 6, pp. 1005-1015, June 2002.

[13] A. Abdi, W. Lau, M.-S. Alouini, and M. Kaveh, "A new simple model for land mobile satellite channels: first- and second-order statistics," IEEE Trans. Wireless Commun., vol. 2, no. 3, pp. 519-528, May 2003.

[14] M. Abramowitz and I. A. Stegun, Handbook of mathematical functions with formulas, graphs, and mathematical tables. 10th ed. U.S. Department of Commerce - N.B.S., Dec. 1972.

[15] A. Erdélyi, W. Magnus, F. Oberhettinger, and F. G. Tricomi, Tables of integral transforms. Vol. I. McGraw-Hill Book Company, Inc., New York-Toronto-London, 1954.

[16] P. W. K. H. M. Srivastava, Multiple Gaussian Hypergeometric Series. John Wiley \& Sons, 1985.

[17] J. F. Paris, "Closed-form expressions for Rician shadowed cumulative distribution function," Electronics Letters, vol. 46, no. 13, pp. 952-953, June 2010.

[18] M. K. Simon and M.-S. Alouini, Digital communication over fading channels. Wiley-IEEE Press, 2005. [Online]. Available: http://www.worldcat.org/isbn/0471649538

[19] F. J. Canete, J. Lopez-Fernandez, C. Garcia-Corrales, A. Sanchez, E. Robles, F. J. Rodrigo, and J. F. Paris, "Measurement and Modeling of Narrowband Channels for Ultrasonic Underwater Communications," Sensors, vol. 16, no. 2, p. 256, 2016. [Online]. Available: http://www.mdpi.com/1424-8220/16/2/256 